\title{
Oxygen Diffusion and Edema With Modern Scleral Rigid Gas Permeable Contact Lenses
}

\author{
Vicente Compañ, ${ }^{1}$ Cristina Oliveira, ${ }^{2}$ Marcel Aguilella-Arzo, ${ }^{3}$ Sergio Mollá, ${ }^{1}$ \\ Sofia C. Peixoto-de-Matos, ${ }^{2}$ and José M. González-Méijome ${ }^{2}$ \\ ${ }^{1}$ Departamento de Termodinámica Aplicada, ETSII, Universidad Politécnica de Valencia, Valencia, Spain \\ ${ }^{2}$ Clinical \& Experimental Optometry Research Lab (CEORLab), Center of Physics (Optometry), School of Sciences, University of \\ Minho, Braga, Portugal \\ ${ }^{3}$ Departamento de Física, Universitat Jaume I, Castellón, Spain
}

Correspondence: José Manuel González-Méijome, Clinical \& Experimental Optometry Research Lab, Department of Physics (Optometry) University of Minho, 4710-057 Braga, Portugal;

jgmeijome@fisica.uminho.pt.

Submitted: January 26, 2014

Accepted: July 29, 2014

Citation: Compañ V, Oliveira C, Aguilella-Arzo M, Mollá S, Peixoto-de-Matos SC, González-Méijome JM. Oxygen diffusion and edema with modern scleral rigid gas permeable contact lenses. Invest Ophthalmol Vis Sci. 2014;55:6421-6429. DOI:10.1167/ iovs.14-14038
Purpose. We defined the theoretical oxygen tension behind modern scleral contact lenses (CLs) made of different rigid gas permeable (RGP) materials, assuming different thickness of the tear layer behind the lens. A second goal was to show clinically the effect of the postlens tear film on corneal swelling.

Methods. We simulated the partial pressure of oxygen across the cornea behind scleral CLs made of different lens materials (oxygen permeability Dk, 75-200 barrer) and different thickness $\left(\mathrm{T}_{\mathrm{av}}, 100-300 \mu \mathrm{m}\right)$. Postlens tear film thicknesses ( $\left.\mathrm{T}_{\text {post-tear }}\right)$ ranging from 150 to 350 $\mu \mathrm{m}$ were considered. Eight healthy subjects were fitted randomly with a scleral lens with a thin and a thick postlens tear layer in two different sessions for a period of 3 hours under open-eye conditions.

Results. The CLs with less than 125 barrer of Dk and a thickness over $200 \mu \mathrm{m}$ depleted the oxygen availability at the lens-cornea interface below $55 \mathrm{~mm} \mathrm{Hg}$ for a postlens tear film of $150 \mu \mathrm{m}$. For a postlens tear film thickness of $350 \mu \mathrm{m}$, no combination of material or lens thickness will meet the criteria of $55 \mathrm{~mm} \mathrm{Hg}$. Our clinical measures of corneal edema showed that this was significantly higher $(P<0.001$, Wilcoxon signed ranks test $)$ with the thicker compared to the thinner $\mathrm{T}_{\text {post-tear }}$ (mean $\pm \mathrm{SD}, 1.66 \pm 1.12$ vs. $4.27 \pm 1.19 \%$ ).

Conclusions. Scleral RGP CLs must be comprised of at least 125 barrer of oxygen permeability and up to $200 \mu \mathrm{m}$ thick to avoid hypoxic effects even under open eye conditions. Postlens tear film layer should be below $150 \mu \mathrm{m}$ to avoid clinically significant edema.

Keywords: scleral lenses, oxygen transmissibility, corneal edema, tear film, boundary layers

\section{$\mathrm{O}$} xygen diffusion models have been applied previously to the cornea ${ }^{1}$ and particularly regarding modern contact lens wear. ${ }^{2-4}$ Scleral lenses have been available from the beginning of contact lens (CL) use. First made of glass, later made of polymethylmethacrylate (PMMA), and now comprising rigid gas permeable (RGP) materials, these devices provided protection and optical regularization of the ocular surface as a result of vaulting without direct contact with the corneal surface. This fitting approach creates a thick postlens tear layer in front of the cornea ${ }^{5}$ and is used currently to compensate several corneal and ocular surface diseases. ${ }^{6,7}$

During the past decades, scleral lenses have been prescribed only for patients with severe ocular surface diseases, ${ }^{8,9}$ However, most RGP manufacturers are now directing their efforts toward developing advanced designs for use in patients with moderate-to-severe corneal distortions and severe ocular surface disease.$^{10}$ Other applications for modern scleral lenses include compensation for high refractive errors, astigmatism among others. ${ }^{7,11}$ Short-term comfort, a protective role in severe ocular surface disease, and the possibility to fit severely distorted corneas without compromising corneal integrity are among the main benefits of such lenses. ${ }^{11}$ Conversely, the thick partially static postlens tear layer creates tear stagnation and the potential hypoxic effects, ${ }^{12}$ which can compromise ocular health over the long term. However, clinical experience and the peer-review literature ${ }^{12,13}$ have confirmed that corneal edema with scleral lenses is not uniform among wearers; there might be interindividual differences in the response to hypoxia as previously reported. ${ }^{14}$ Further, the fitting characteristics of these devices also may have an important role. Despite recent publications that have suggested the importance of the postlens tear film thickness and its limited oxygen permeability as one cause, ${ }^{15}$ to our knowledge no previous study has presented theoretical simulations with clinical evidence of such potential effect. Thick tear layers are necessary to support larger lenses $(<18 \mathrm{~mm})$, while smaller lenses $(15-18 \mathrm{~mm})$ are associated with a thinner tear thickness. Given the expanding role of large diameter lenses and scleral lenses in managing severely distorted corneas and the particular physiological conditions they create on the cornea, it is necessary to evaluate the impact that these devices exert regarding hypoxia. It is of particular interest to evaluate the impact of thick tear film underneath these lenses, which is a unique figure of these fittings considering the limited permeability of water ( 99 barrers) that creates additional resistance to oxygen diffusion to the cornea, similar to the conditions created in a piggyback system. ${ }^{16,17}$ However, while in piggyback systems some tear exchange exists underneath the lenses, this seems to be very limited, if 
existing, after lens settling, with scleral lenses. The water permeability is the product between the diffusion coefficient for oxygen in water at $35^{\circ} \mathrm{C}\left(D=3 \times 10^{-5} \mathrm{~cm}^{2} / \mathrm{s}\right)^{18}$ and the oxygen solubility coefficient in water $k=3.3 \times 10^{-6} \mathrm{~cm}^{3}$ of $\mathrm{O}_{2} /$ $\mathrm{mL}$ of water/mm $\mathrm{Hg}){ }^{19}$ In the current study, a three-layer physical model is used to quantify the oxygen transport through the cornea-prelens tear film-lens system. We assumed that the three layers were flat. The cornea is thin $(531.5 \mu \mathrm{m})$ compared to its lateral dimension (approximately 10,000 $\mu \mathrm{m}$ ), and the lateral diffusion along the cornea is negligible compared to that under normal diffusion conditions.

The goal of this theoretical study was to apply the simple metabolic oxygen diffusion model described by Chhabra et al. $^{20}$ to different situations with varying lens oxygen permeability (Dk), central lens thickness $\left(T_{c}\right)$, and postlens tear film thickness $\left(\mathrm{T}_{\text {post-tear }}\right)$, and verify the theoretical predictions by measuring the corneal edema after 3 hours of scleral CL wear with different sagittal depths to create different posttear film layers.

\section{MeTHODS}

\section{Theoretical Modelization}

Anatomic and Physiological Parameters. Different investigators have considered different parameters to create their models of oxygen depletion across the cornea behind CLs. In the current study, we made additional efforts to establish those parameters most commonly accepted considering the classical and most recent literature. We also assumed that the system integrates a relatively thick CL with a thick postlens tear film thickness. The Table shows the values and units of the parameters considered for the lens, postlens tear film thicknesses, and corneal thicknesses. We did not consider the prelens tear film layer because of its negligible contribution to the system considering the much thicker lens and postlens tear film. Other parameters, such as oxygen consumption, partial pressure of oxygen in the front ocular surface at sea level, and the aqueous humour, also are reported. While most parameters have been accepted consistently in the literature, others have not. The values of human corneal oxygen flux have been reported in the literature to range widely from 1.6 to 10.9 $\mu \mathrm{L} \cdot \mathrm{cm}^{-2} \cdot \mathrm{h}^{-1}{ }^{2}$ We have considered a value of $531.5 \mu \mathrm{m}$ for corneal thickness based on the meta-analysis conducted by Doughty and Zaman ${ }^{21}$ instead of the various values considered in previous studies of oxygen consumption.

Since oxygen diffusivity and consumption in the human cornea have not been measured directly, Larrea et al., ${ }^{22}$ Alvord et al., ${ }^{23}$ and Chhabra et al. ${ }^{20}$ have proposed mathematical models of time-dependent oxygen diffusion that allow estimation of corneal consumption and diffusion. Such models use nonlinear Monod kinetics to describe the local oxygenconsumption rate. Nevertheless, although oxygen consumption is a result of corneal cell metabolism that depends on a great number of factors, Chhabra et al. ${ }^{20}$ assumed that the oxygen consumption only depends on the partial pressure of oxygen and used parameters $Q_{c, \max },(D k)_{c}$ and $K_{m}$, where $\mathrm{Q}_{\mathrm{c} \text {,max }}$ is the maximal corneal oxygen-consumption rate, $\mathrm{k}_{\mathrm{c}}$ is the corneal oxygen solubility, $D_{c}$ is the corneal oxygen diffusion coefficient, and $\mathrm{K}_{\mathrm{m}}$ is a constant that determines the shape of the $Q$ versus $p_{c}$ curve and represents the oxygen pressure when the aerobic metabolism in the cornea reaches maximal oxygen consumption. ${ }^{20,24}$

In the current study, we evaluated the impact of considering the Monod kinetics model on the cornea/postlens tear film/ lens system described previously for scleral CLs in which the postlens tear film is very thick and its resistance cannot be considerable negligible. To obtain the oxygen pressure profile in the cornea we considered the values of parameters $Q_{c, \max }$ and $\mathrm{K}_{\mathrm{m}}$ reported by Chhabra et al. ${ }^{20}$ The Table shows the different values for the parameters used in the numerical solution of the equations.

A value of $24 \mathrm{~mm} \mathrm{Hg}$ was considered for the aqueous humour partial pressure of oxygen as reported in a recent study $^{25}$ instead of the value of $55 \mathrm{~mm} \mathrm{Hg}$ assumed in the classical literature ${ }^{1}$ and according values recently considered in this field. ${ }^{24,26}$

Lens Parameters Entered Into the Model. Assuming known values of partial pressure at the front lens surface under open and closed eye conditions, the partial pressure of oxygen at the aqueous and oxygen consumption in endothelium, epithelium, and stroma, the oxygen partial pressure distribution profiles across the contact lens-tear film-corneal system were calculated (Fig. 1). Lens Dk was allowed to have values of $75,100,125,150,175$, and 200 barrers with thicknesses of 100 to $300 \mu \mathrm{m}$ in $50-\mu \mathrm{m}$ steps. The postlens tear film was assumed to change between 150 and $350 \mu \mathrm{m}$ in $50-\mu \mathrm{m}$ steps. The resistance to oxygen flux is negligible at the front surface of the RGP material because the prelens tear films is very thin (2$5 \mu \mathrm{m})^{27}$ compared to the resistance produced by the lens $(100-300 \mu \mathrm{m})$ and the postlens tear film layer (tear film layer between $150-350 \mu \mathrm{m}$ ) in contact with the cornea when a scleral lens is worn. This value has not been taken into account in the calculations. The theoretical development is available in the Appendix. This model applies only in the area of the thicker fluid layer (i.e., the central cornea). Because the fluid layer is thinner over the limbus, or above the ecstatic area, different results would be found in those areas.

\section{Clinical Trial}

Eight right eyes of eight young healthy patients (six women, two men; $22 \pm 4$ years) were fitted with a $15.5-\mathrm{mm}$ corneoscleral lens made of a gas permeable fluorsilicone acrylate (paflufocon D, Dk, 100 barrers). All volunteers received full information about the study purpose and procedures, and signed a consent form before being enrolled in the study. The study was reviewed and approved by the Ethics Committee of the School of Sciences at the University of Minho. Lenses were fitted to create two significantly different postlens tear film thickness at the optical axis of the cornea (shallower, $150 \mu \mathrm{m}$; deeper, $350 \mu \mathrm{m}$ ). The predicted vaulting of the lens over the central cornea was estimated using the sagittal corneal depth derived from the Medmont E300 at a chord diameter of $10.0 \mathrm{~mm}$ as recommended by the lens distributor (Lenticon, Madrid, Spain). At the preliminary visit, the lenses were fitted based on corneal topography and tested to warrant appropriate fitting and wearing comfort. The volunteers were scheduled for two additional visits separated by 1 week. Deeper and shallower fitting trials were assigned randomly to visits 1 and 2 . The central lens thickness of each lens used was measured with a Redher thickness gauge (Redher Development Co., Castro Valley, CA, USA). This parameter was used as a comparator to estimate the separation between the back of the lens and the anterior corneal surface with an optical section in the slit-lamp following the habitual clinical method to guess the separation in clinical routine.

Subjects were required to attend each visit at the same time in the morning after having been awake for at least 2 hours. Approximately $300 \mu \mathrm{L}$ of sterile saline (Lens Plus; Abbott Medical Optics, Inc., Santa Ana, CA, USA) was used to fill the internal reservoir between the lens and cornea. At each visit, the central corneal thickness (CCT) and corneal topography were obtained before lens insertion. One corneal topography measurement (Medmont E300 Corneal Topographer; Medmont 
TABLE. Parameters Considered in the Model to Obtain the Partial Pressure of Oxygen at Different Depths Into the Cornea

\begin{tabular}{|c|c|c|c|}
\hline Parameter & Symbol & Value & Units \\
\hline Atmospheric partial pressure of oxygen under open eye conditions & $p_{t c}$ & 155 & $\mathrm{~mm} \mathrm{Hg}$ \\
\hline Aqueous partial pressure of oxygen & $p_{t c}$ & 24 & $\mathrm{~mm} \mathrm{Hg}$ \\
\hline Palpebral conjunctiva oxygen pressure & $p_{p c}$ & 61.5 & $\mathrm{~mm} \mathrm{Hg}$ \\
\hline Corneal oxygen consumption & $\mathrm{Q}$ & $1.05 \cdot \mathrm{E}^{-04}$ & $\mathrm{~cm}^{3}\left(\mathrm{O}_{2}\right) \cdot \mathrm{cm}^{-3} \cdot \mathrm{s}^{-1}$ \\
\hline Corneal permeability & $\mathrm{Dk}_{\text {cornea }}$ & 86 & Barrers \\
\hline Central corneal thickness & CCT & 531.5 & $\mu \mathrm{m}$ \\
\hline Epithelium & $\mathrm{T}_{\mathrm{ep}}$ & 50 & $\mu \mathrm{m}$ \\
\hline Stroma & $\mathrm{T}_{\mathrm{st}}$ & 480 & $\mu \mathrm{m}$ \\
\hline Endothelium & $\mathrm{T}_{\mathrm{en}}$ & 1.5 & $\mu \mathrm{m}$ \\
\hline Water permeability* & $\mathrm{Dk}_{\mathrm{water}}$ & 99 & Barrers \\
\hline Postlens tear film thickness & $\mathrm{T}_{\text {post-tear }}$ & $150-350$ & $\mu \mathrm{m}$ \\
\hline Lens permeability & $\mathrm{Dk}_{\text {lens }}$ & $75-200 \dagger$ & Barrers \\
\hline Lens thickness, central & $\mathrm{T}_{\mathrm{c}}$ & $100-300 \ddagger$ & $\mu \mathrm{m}$ \\
\hline
\end{tabular}

$D k$ units (barrers) $=10^{-11}\left(\mathrm{~cm}^{2} / \mathrm{s}\right)\left(\mathrm{mL} \mathrm{O}_{2} \cdot \mathrm{mL}^{-1} \cdot \mathrm{mm} \mathrm{Hg}^{-1}\right) ; D k / t_{a v}$ units (barrers $\left./ \mathrm{cm}\right)=10^{-09}(\mathrm{~cm} \mathrm{~mL} \mathrm{O}) /(\mathrm{mL} / \mathrm{s} \mathrm{mm} \mathrm{Hg}) ; D_{c}=2.81 \times 10^{-5}$ $\mathrm{cm}^{2} / \mathrm{s} ; k_{c}=3.06 \times 10^{-5} \mathrm{~cm}^{3}$ of $\mathrm{O}_{2}(\mathrm{sTP}) / \mathrm{cm}^{3}$ of tissue $/ \mathrm{mm} \mathrm{Hg}$.

${ }^{*} D_{w}=3 \times 10^{-5} \mathrm{~cm}^{2} / \mathrm{s} ; k_{w}=3.3 \times 10^{-5} \mathrm{~cm}^{3}$ of $\mathrm{O}_{2}(\mathrm{sTP}) / \mathrm{cm}^{3}$ of tissue $/ \mathrm{mm} \mathrm{Hg}$

† Currently the highest permeability for these materials is approximately 163 barrer.

‡ The thinnest designs available in the market are approximately $150 \mu \mathrm{m}$ at minimum for larger diameter $(>15 \mathrm{~mm})$ rigid gas permeable lenses.

Pty., Nunawading, Australia) and 5 repeated measures of CCT were obtained (Handyscan 100; Tomey Co., Nagoya, Japan). Pachymetry was measured after instillation of a topical anesthetic (oxybuprocaine 0.5\%; Anestocil, Laboratórios, Edol, Portugal). The swelling response was calculated according to Equation 1. Anesthetic drops are known to increase epithelial permeability. ${ }^{28}$ This could change the thickness of the corneal stroma by osmotic pressure. However, any systematic effect probably would cancel when deriving the percent thickness change between visits as the same procedure was followed.

$$
\text { Edema }(\%)=(\text { Final CCT }- \text { Baseline CCT } \times 100 /
$$

(Baseline CCT)
The contralateral eye acted as control in all visits. Lenses were worn for 3 hours under open-eye conditions, and then the same parameters were measured immediately after lens removal. Within 1 minute after application of the anesthetic, corneal topography was measured first to avoid distortion of the corneal surface by the pachymetric probe. One high quality measure according to the automatic focusing and centration system of the topographer was obtained to make sure that the anterior corneal surface did not suffer any distortion as a result of lens wear. If dry spots or irregularities were detected, the capture was repeated. The patient was requested to blink before capture to ensure uniform spread of tears over the corneal surface. Any molding effect induced by central lens pressure to the cornea can affect assessment of

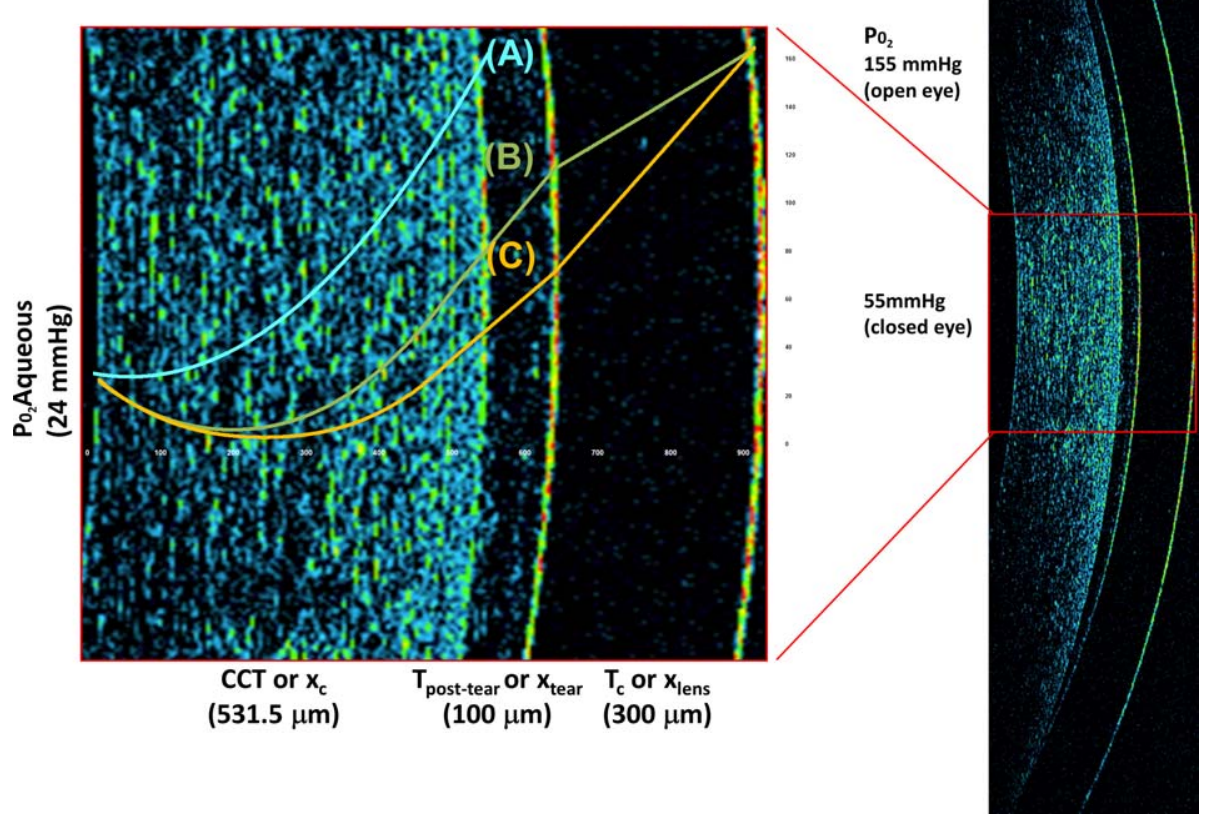

Figure 1. Distribution of oxygen across the lens, tear film, and corneal thickness under conditions of no lens wear (A), wearing of a contact lens with $300 \mu \mathrm{m}$ of central thickness, 200 barrers of oxygen permeability with a $150-\mu \mathrm{m}$ postlens central tear film thickness under open-eye conditions (B), and the same conditions for a 75 barrers lens (C). 

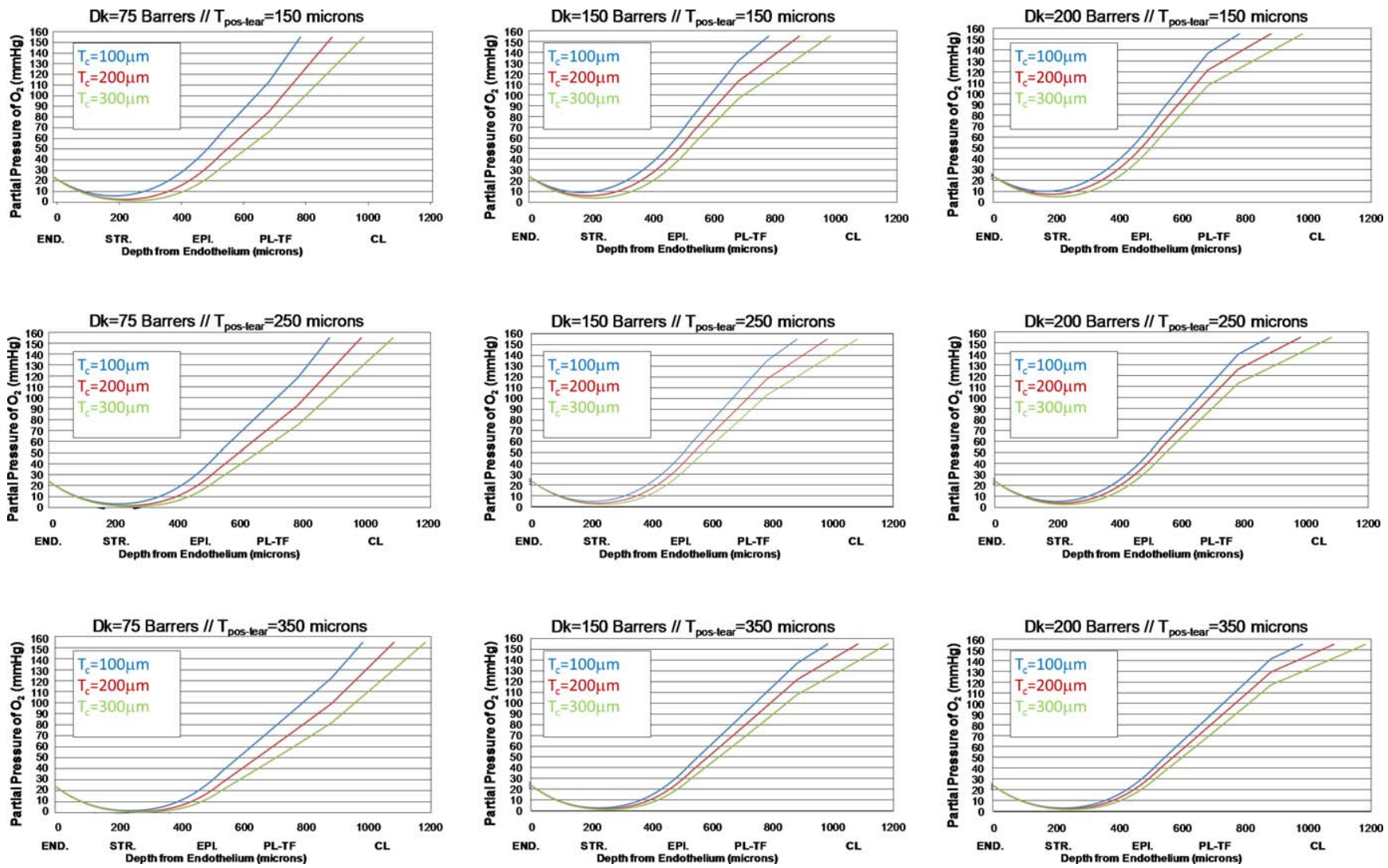

FIGURE 2. Distribution of the partial pressure of oxygen profiles across the lens, postlens tear film, and corneal thickness for different combinations of materials $\left(D k=75,150\right.$, and 200 barrer, left to right), lens thickness $\left(T_{c}=100,200\right.$, and $300 \mu \mathrm{m}$, within each graph) and tear thickness $\left(T_{p o s t-t e a r}\right.$ $=150,250$, and $350 \mu \mathrm{m}$, top to bottom). END, endothelium; STR, stroma; EPI, epithelium; PL-TF, postlens tear film.

edematous response. The average apical curvature and simulated keratometric readings were used to evaluate any potential changes. Immediately after corneal topography, five pachymetry readings were obtained at the center of the cornea. The probe was applied over the corneal reflex of a light source placed perpendicular to the measured eye, while the other eye was occluded to avoid fixation deviations. Pachymetric data were recorded as the average of the five measurements if the standard deviation was inferior to $5 \mu \mathrm{m}$ from the five consecutive readings. The lens-to-cornea separation was estimated by comparing the actual central lens thickness with the thickness of the postlens tear film as in clinical routine.

Statistical analysis was performed using SPSS v.21 (IBM, Chicago, IL, USA) to assess the differences in corneal curvature and thickness before and after each lens fitting trial, between lens wearing eye and control eye, and between different lenses worn in visits 1 and 2 . Considering the reduced size of the sample, the nonparametric Wilcoxon signed rank test was used to compare the edematous response between both vaulting strategies

\section{Results}

\section{Theoretical Simulation}

Figure 2 shows the partial pressure of oxygen profiles across the lens, postlens tear film, and cornea for different combinations of lens materials, lens thicknesses, and tear film thicknesses. Three regions of oxygen depletion were seen across the lens-tear-cornea system. In the lens and tears, the depletion was linear as predicted by the Fick's law, because no oxygen consumption is present at that level. The slopes for oxygen depletion in the lens differ as a function of the oxygen permeability of the material. Instead, the metabolic activity of the cornea and the supply of oxygen from the anterior chamber in contact with the endothelium defines the shape of the depletion curves across the cornea.

We observed that for lower Dk values, the distribution of oxygen depended strongly on lens and tear film layer, while for the highest Dk value, the partial pressure of oxygen at the corneal surface is almost independent of postlens tear film layer. Thus, for a 75 -barrer lens with a $150-\mu \mathrm{m}$ postlens tear film, the partial pressure of oxygen in contact with the epithelium is $65 \mathrm{~mm} \mathrm{Hg}$ in the unlikely event of a $100-\mu \mathrm{m}$ lens. However, for a $300-\mu \mathrm{m}$ lens, which is relatively common in large diameter RGP CLs, this would be $33 \mathrm{~mm} \mathrm{Hg}$.

If we compared the effect of the postlens tear thickness, the partial pressure of oxygen in contact with the epithelium would be $65 \mathrm{~mm} \mathrm{Hg}$ or $37.3 \mathrm{~mm} \mathrm{Hg}$ for a 200- $\mu \mathrm{m}$ lens with $150-$ or $350-\mu \mathrm{m}$ postlens tear film, respectively.

For lenses as thick as $300 \mu \mathrm{m}$ and tear reservoir thickness over $250 \mu \mathrm{m}$, some degree of anoxia is expected in the middleto-deep stroma for lenses with a Dk below 150 barrer.

However, for cases in which the oxygen permeability of the lens materials was 75 barrers or below and postlens tear film thickness thicker than $200 \mu \mathrm{m}$, the model predicts zero oxygen availability at several locations across the corneal depth at the optical center of the cornea.

Figure 3 summarizes the physiologic conditions behind the scleral lens for different $\mathrm{Dk}$ values and different lens thicknesses. With lenses with lower Dk lenses, the partial pressure in contact with the epithelium changed from 65 to 33 

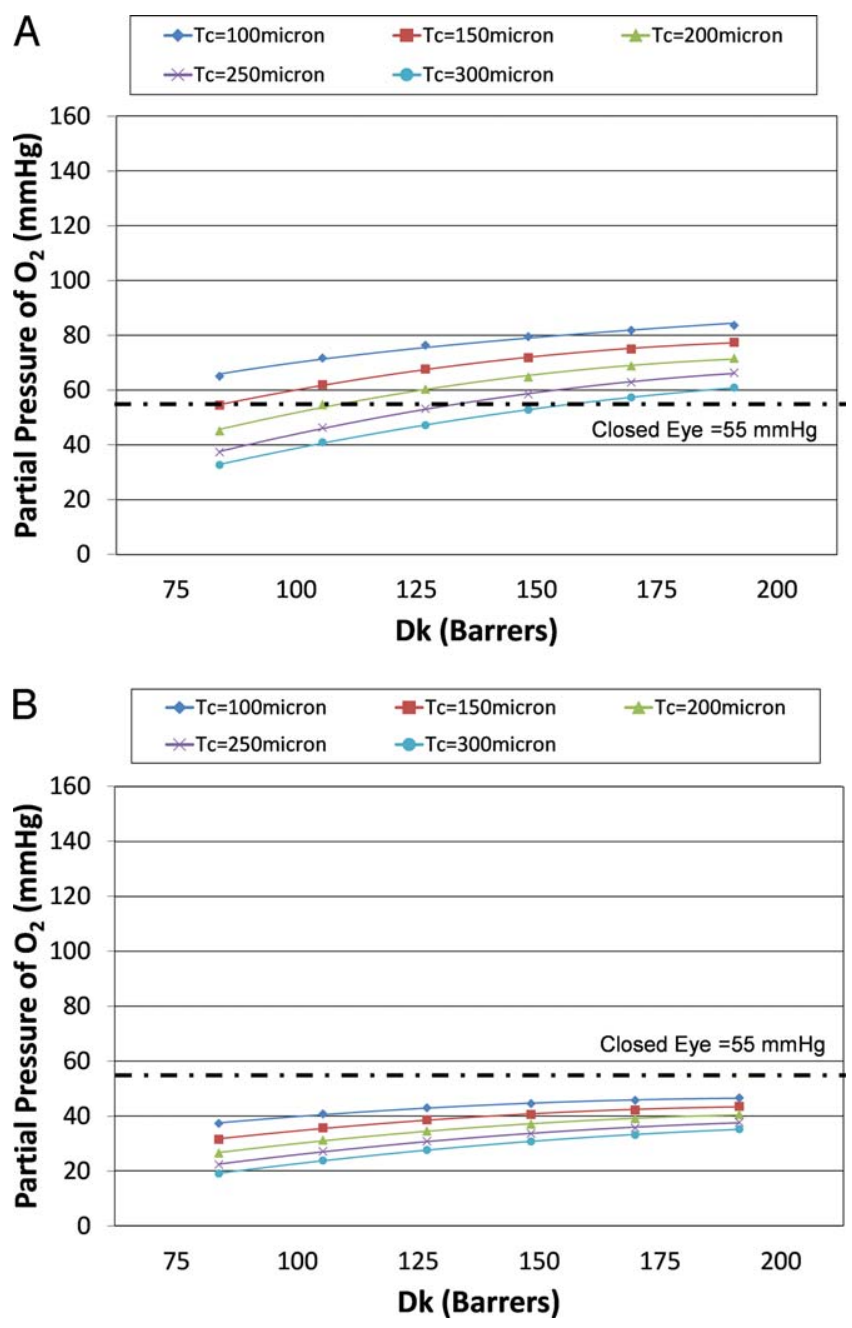

Figure 3. (A) Partial pressure of oxygen at the corneal surface behind lenses of different thickness $(T c=100,150,200,250$, and $300 \mu \mathrm{m})$ considering a "thin" tear thickness of $150 \mu \mathrm{m}$ and (B) considering a "thick" tear thickness of $350 \mu \mathrm{m}$.

$\mathrm{mm} \mathrm{Hg}$ when the lens thickness changed from 100 to $300 \mu \mathrm{m}$ for a $150-\mu \mathrm{m}$ postlens tear film. Instead, for a $150-\mu \mathrm{m}$ lens with a $150-\mu \mathrm{m}$ postlens tear film, increasing the permeability from 75 to 200 barrers changed the partial pressure of oxygen in contact with the epithelium from 65 to $83.6 \mathrm{~mm} \mathrm{Hg}$, respectively. Varying the postlens tear thickness created a more significant effect on oxygen available to the cornea. Thus, for a $100-\mu \mathrm{m}$ thick lens made of a 175-barrer material, an increase from 150 to $350 \mu \mathrm{m}$ in postlens tear film thickness decreased the partial pressure of oxygen in contact with the epithelium from 83 to $46 \mathrm{~mm} \mathrm{Hg}$

Considering a minimal desirable partial pressure of oxygen at the corneal surface similar to those of the closed eye as a criterion, all simulations "on average" satisfied these conditions (Fig. 3A). It is well known that closed eye conditions induce the so called physiological edema of approximately $4 \%$. However, when we consider the worst scenario of maximum tear film thickness (Fig. 3B), the material must have at least 125 barrer of oxygen permeability so that even thicker lenses (250 and $300 \mu \mathrm{m}$ ) can satisfy the criterion. Our simulations predicted that none of the combinations provided to the cornea enough oxygen to avoid hypoxic effects.

Concerning the role of lens and tear film thickness, as expected, the relative importance of the tear film layer

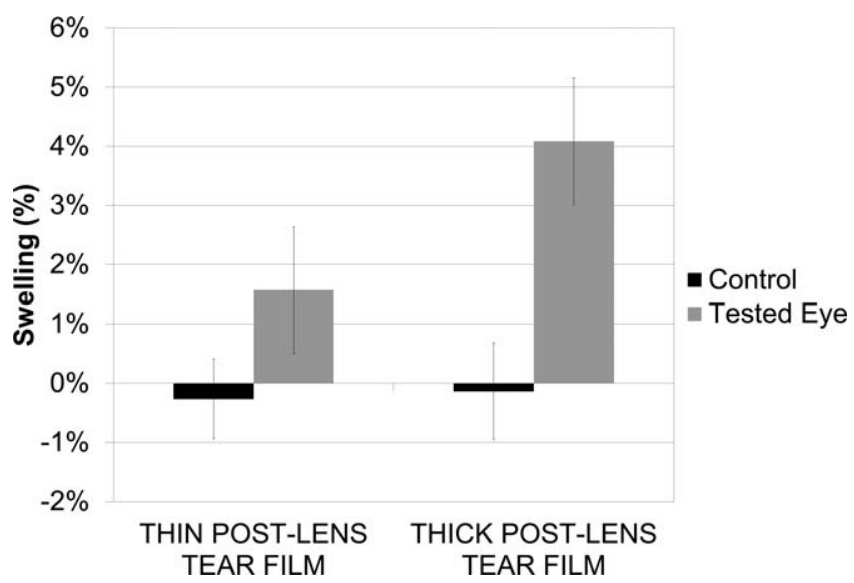

Figure 4. The mean and standard deviation values of corneal swelling induced by two different scleral lens fitting in a cohort of eight subjects fitted randomly with a postlens tear film thickness of $150 \mu \mathrm{m}$ and with a postlens tear film thickness of $350 \mu \mathrm{m}$.

thickness is greater for lenses of lower oxygen permeability. A $100-\mu \mathrm{m}$ increase in lens thickness reduced the partial pressure of oxygen at the corneal surface between $17 \%$ and $21 \%$ for a 75 -barrer material and approximately $6 \%$ for a $200-$ barrer material. The same increase in tear film thickness had an impact of $14 \%$ to $16 \%$ in the partial pressure of oxygen at the corneal surface for a 75 -barrer material and approximately $12 \%$ to $14 \%$ for a 200 -barrer material.

\section{Clinical Trial}

The thickness of the postlens tear film calculated theoretically by considering the difference between the corneal sagittal depth obtained before lens fitting with the Medmont E300 and the lens sagittal depth was estimated at $60 \pm 28 \mu \mathrm{m}$ for the shallower fitting and $267 \pm 54 \mu \mathrm{m}$ for the deeper fitting $(P=$ 0.012 , Wilcoxon signed ranks test). No significant $(P>0.05$, Wilcoxon signed ranks test) differences were observed clinically in corneal topography in eyes wearing lenses or in contralateral eyes at both visits based on the apical radius and simulated keratometry estimations. The initial vault of the lenses was higher than the vault after 3 hours of lens wear; however, no lens pressure on the cornea was observed after 3 hours of lens wear. The tear layer separation underneath the lens at the optical center of the cornea was in all cases at least half the thickness of the lens in the shallower fitting strategy and 1.3 times the thickness of the lens in the deeper vaulting fitting strategy.

The corneal thickness increased significantly with both lenses. The median value of the swelling response was $1.59 \%$ with the thinner postlens tear film and $3.86 \%$ with the thicker postlens tear film. The corneal thickness changes were statistically significant between eyes wearing lenses and control eyes at both visits $(P<0.023$, Wilcoxon signed ranks test) and between both visits for the same eye with the deeper tear reservoir inducing the highest value of swelling $(P<$ 0.001 , Wilcoxon signed ranks test). The average and standard deviation of the CCT changes with both lenses is shown in Figure 4 .

\section{Discussion}

Despite high Dk of the modern RGP materials, the significantly thicker tear layer created behind scleral lenses increased significantly the effect of boundary layers, including a low-to- 


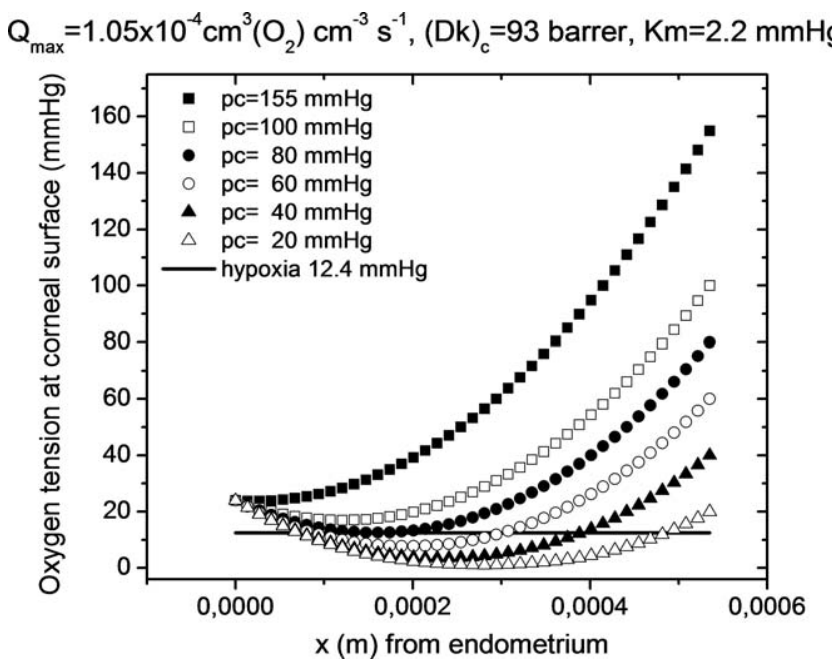

Figure 5. Oxygen distribution across the cornea derived from Equation 1 in the Appendix considering the rate of oxygen consumption for Monod kinetics model from Chhabra et al., ${ }^{20}$ assuming a constant $k=2.2 \mathrm{~mm} \mathrm{Hg}$, for different partial pressures of oxygen at the epithelial surface.

moderate level of edema. This might be related with the limited availability of oxygen to the cornea as predicted by our theoretical models. The current study modeled the unidimensional distribution of oxygen across the cornea and has proved that, as expected, an increased edematous response confirms the presence of a hypoxic situation within the cornea for the thicker tear film layers. Despite the edema found within physiological levels (an average swelling of $4 \%$ for the thicker tear film thickness), this is approximately equivalent to have the cornea during waking hours under the same physiologic hypoxia included by closed eye conditions. The clinician should bear in mind that a thinner postlens tear film will be the best choice, particularly when fitting the compromised cornea. This approach certainly will minimize the chronic hypoxic effects induced to the cornea during waking hours, which will add up to the physiologic hypoxia that affects the cornea overnight as a result of lid closure.

When designing these experiments, there was some difficulty choosing the correct values for parameters, such as corneal oxygen consumption, corneal and water permeability, and partial pressure of oxygen at the different interfaces. During the last decades, there have been consistent criteria for parameters, such as the partial pressure of oxygen at the sea level under open-eye $(155 \mathrm{~mm} \mathrm{Hg})$ and closed-eye conditions from the palpebral conjunctiva $(55 \mathrm{~mm} \mathrm{Hg}$ ). However, other parameters, such as the partial pressure of oxygen at the endothelial corneal surface $(55 \mathrm{~mm} \mathrm{Hg}$ ), had been assumed during years, although recent values of 24 to $24.1 \mathrm{~mm} \mathrm{Hg}$ have been considered theoretically or measured clinically, ${ }^{24,25}$ respectively. Other parameters, such as the endothelium, stroma, and epithelium thickness, have varied greatly in the literature depending on the methods of measurement, among other factors. ${ }^{21,29,30}$

The value of the water permeability used in this study ( $P=$ $\mathrm{D}_{\mathrm{w}} \mathrm{k}_{\mathrm{w}}$ ) was 99 barrers, that is, the product between the diffusion coefficient for oxygen in water at $35^{\circ} \mathrm{C}\left(D=3 \times 10^{-5}\right.$ $\left.\mathrm{cm}^{2} / \mathrm{s}\right)^{18}$ and the oxygen solubility coefficient in water $k=3.3$ $\times 10^{-6} \mathrm{~cm}^{3}$ of $\mathrm{O}_{2} / \mathrm{mL}$ of water $/ \mathrm{mm} \mathrm{Hg}$ ). ${ }^{19}$

The current results agreed with the preliminary estimations of Weissman and $\mathrm{Ye}^{17}$ who predicted a partial pressure of oxygen of $60 \mathrm{~mm} \mathrm{Hg}$ at the corneal surface beneath a $400-\mu \mathrm{m}$ thick lens with Dk of 100 barrers with a tear reservoir of $200 \mu \mathrm{m}$.

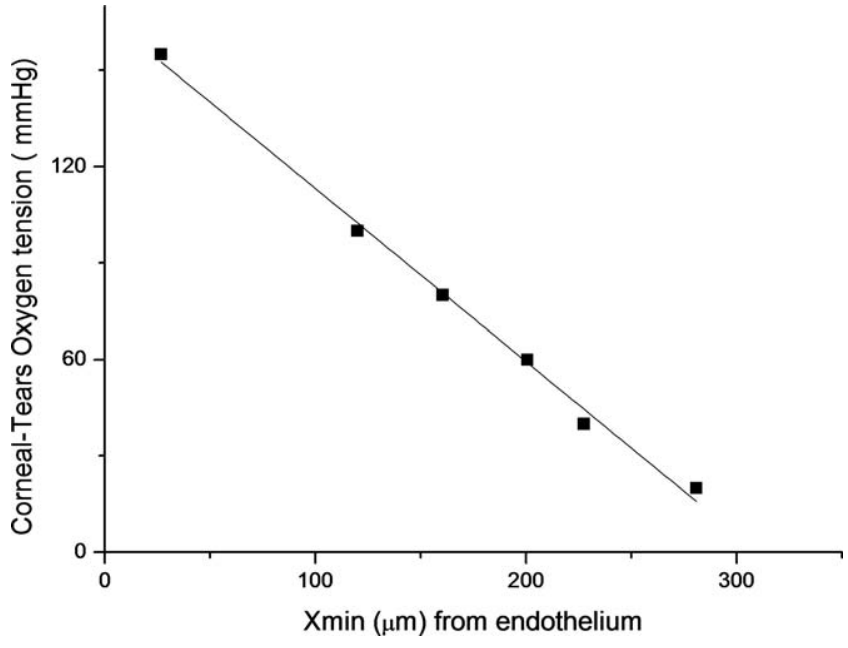

FIGURE 6. Variations in the position of the minimal oxygen availability $\left(\mathrm{X}_{\min }\right)$ as a function of the oxygen tension at the cornea-tear film interface using the Monod kinetics model. ${ }^{20}$

The current study predicted some degree of corneal hypoxia and even anoxia under different circumstances that are compatible with current scleral lens fitting. Further, our clinical results showed a significantly higher edematous response, although it was within physiological limits, when a thicker postlens tear film was present. If lower Dk values are to be used, the corneal health might be compromised over the long term. A possible strategy might be the removal of the lens after several hours to refill the postlens tear film reservoir. Other strategies imply the use of smaller lenses when possible, and/or reduction of the tear thickness and/or selection of a higher permeable material.

Classical models used consider that the oxygen consumption across the cornea is constant. However, more recently the Monod kinetics model has been proposed to define oxygen availability across the cornea. ${ }^{20,31}$ Figure 5 simulates the oxygen distribution across the cornea for different values of partial pressure at the epithelial surface from $155 \mathrm{~mm} \mathrm{Hg}$ (normal open-eye conditions) to $20 \mathrm{~mm} \mathrm{Hg}$ according to the Monod kinetics model with $k=2.2 \mathrm{~mm} \mathrm{Hg}$.

Figure 5 shows that the oxygen distribution into the cornea does not produce negative values for the partial pressure of oxygen; thus, avoiding the physical impossibility of the oxygen pressure falling below zero. The shift toward the middle stroma of the minimally observed oxygen tension $\left(\mathrm{X}_{\min }\right)$ also was verified using this model as the oxygen tension at the cornea-tear film interface decreased.

In Figure 6, we plotted the position of the $\mathrm{X}_{\min }$ with its respective oxygen tension for different partial pressures of oxygen at the cornea-tear film interface. This plot shows a nearly linear correlation between the position of minimal oxygen availability $(\mu \mathrm{m})$ and the oxygen tension $(\mathrm{mm} \mathrm{Hg})$ at the corneal-tear film interface using the Monod kinetics model with $k=2.2 \mathrm{~mm} \mathrm{Hg}$. According to this theoretical calculation, the position of minimum oxygen availability across the corneal depth displaces toward the endothelium as the corneal-tear film oxygen tension is depleted due to contact lens-induced hypoxia.

Several conclusions were drawn from this study. Importantly, material selection seems to be critical for maintaining corneal health under a mini-scleral or sclera lens once tear film turnover is virtually absent. To guarantee that there is sufficient oxygen to reach the cornea in the worst scenario (maximal 
lens thickness and maximal postlens tear film thickness), the oxygen permeability must at least 150 barrers.

Our clinical verification had some limitations. One was that the lenses tested had different optical powers and, thus, different oxygen transmissibility values. However, the power change in the lens is not expected to produce a significant physiological situation as it will require a large change in negative power of the lens to produce a significantly different oxygen transmissibility. Another limitation was the fact that no objective measures of the postlens tear film layer have been obtained. Considering that the lenses were fitted based only on corneal topography data, it was expected that the unknown perilimbal scleral area might affect the final clearance of the lens in a relatively unpredictable way. To partially overcome this, we clinically observed with a slit-lamp the postlens tear reservoir during the study using an optical section and compared this to the central thickness of the lens previously measured. Although we cannot report the values of the final separation at a micron resolution, clinical observation is the common practice that allows us to confirm that the tear thickness was similar to the lens thickness that varied between 180 and 250 depending on the power of the lenses used. Clinical observation showed that the thickness of the postlens tear film was approximately one-third of the central lens thickness in the case of the thinner postlens tear film fitting and approximately the same thickness in the case of the thicker postlens tear film, which agreed with the theoretical estimations based on corneal topography and CL sagittal depth. This limitation is minimized by the fact that the same clinician obtained all the clinical measures, which makes the process more reproducible. A third limitation was the small sample of healthy subjects. Future systematic studies with larger samples should be conducted to measure objectively the lens clearance with a noninvasive system that simultaneously measures the corneal thickness with the lens on the eye. This might be accomplished using optical coherence tomography.

Different studies have correlated the oxygen transmissibility of $\mathrm{CL}$ and the edematous response of the cornea, ${ }^{32}$ which also is the basis for determining the critical oxygenation needed to avoid corneal hypoxia. ${ }^{33,34}$ Given those relationships and the estimations reported in the present study, we expect values of edema above those reported by Pullum and Stapleton. ${ }^{12}$ They measured 3\% edema response after 3 hours of wear of a 115barrer, $600-\mu \mathrm{m}$ thick scleral lens, which is approximately the physiological edema induced overnight, ${ }^{35}$ with the eye exposed to a partial pressure of oxygen of $55 \mathrm{~mm} \mathrm{Hg}$ from the tarsal conjunctiva. Although those investigators did not report the thickness of the tear reservoir, a 3\% edema response seems to be consistent with the calculations of our model that will predict a partial pressure of oxygen below $45 \mathrm{~mm} \mathrm{Hg} \mathrm{(1/3}$ of normal availability under open-eye conditions, and similar to closed-eye conditions) considering a $200-\mu \mathrm{m}$ tear reservoir. Tear mixing beneath the scleral lens was not considered in the current calculations; steady-state conditions were considered. Although we can speculate that tear exchange could occur, resulting in higher oxygen availability than might be expected through lens transmissibility, this is not a likely scenario considering the clinical observations. Currently, to our knowledge there is no objective evidence of the absence of tear exchange behind scleral CLs. However, a common clinical observation is that scleral lenses become almost static minutes after insertion as they rest in the soft bulbar conjunctiva. So, no tear exchange is expected after the lens is stabilized on the conjunctiva. The current results also should be considered with caution in diseased eyes. The postlens tear profile also might change significantly in ectatic corneas. Even for normal eyes and for ocular disease related to severe dry eye, the postlens tear film profile is not uniform and the calculations presented may not apply to any local point of the cornea. Our simulations and clinical measures have been referred to the optical center of the cornea. Despite the limited edematous response found, it is not expected to cause an adverse physiologic corneal response in the short term. The long-term effects of chronic physiological hypoxia with scleral lenses must be evaluated further, and strategies to minimize the hypoxic conditions during the waking hours should be considered by clinicians as previously discussed. However, we do not know the behavior of such response in the diseased ocular surface where these lenses usually are applied. Regarding normal healthy eyes, the thickness of the postlens tear reservoir affects the level of edematous response under open-eye conditions, but this remains at the physiological level for most subjects evaluated.

In summary, for the thinner postlens tear film scenario (100 $\mu \mathrm{m})$, any combination of lenses with a Dk above 75 barrers and a lens thickness up to $300 \mu \mathrm{m}$ should provide the anterior corneal surface with a partial pressure of oxygen that is above the amount available for the naked cornea under closed-eye conditions. For the thicker tear reservoir $\left(\mathrm{T}_{\text {post-tear }}=350 \mu \mathrm{m}\right)$, it is expected that the partial pressure of oxygen will be under this level for thicker lenses with permeability below 125 barrer.

\section{Acknowledgments}

Presented partly at the annual meeting of the Association for Research in Vision and Ophthalmology, Fort Lauderdale, Florida, United States, May 7, 2012.

Supported by the Ministerio de Educación y Ciencia (MEC) through Project ENE2011-24761, and by FEDER through the COMPETE Program and by the Portuguese Foundation for Science and Technology (FCT) in the framework of the Strategic Project PEST-C/FIS/UI607/2011. The authors alone are responsible for the content and writing of the paper.

Disclosure: V. Compañ, None; C. Oliveira, None; M. AguilellaArzo, None; S. Mollá, None; S.C. Peixoto-de-Matos, None; J.M. González-Méijome, None

\section{APPENDiX}

\section{Multilayer Slab Model to Study Oxygen Distribution}

The non-steady state diffusion equation that gives oxygen tension as a function of time and position for homogeneous slab of oxygen-consuming tissue (assuming a one-dimensional model for the cornea) is given by

$$
\frac{\partial^{2} p_{c}}{\partial x^{2}}-\left(\frac{Q}{D k}\right)_{c}=\frac{1}{D} \frac{\partial p_{c}}{\partial t},
$$

where $p_{c}(x)$ is the partial pressure of oxygen in the cornea, $D$ is the diffusion coefficient of oxygen in the tissue $\left(\mathrm{cm}^{2} / \mathrm{sec}\right), k$ is the oxygen solubility coefficient in the cornea tissue (i.e., Henry's law constant) in $\left(\mathrm{cm}^{3}\right.$ of $\mathrm{O}_{2} / \mathrm{cm}^{3}$ layer $/ \mathrm{mm}$ of $\mathrm{Hg}$ ), and $x$ is the distance perpendicular to the surface in $(\mathrm{cm}) ; Q$ is the oxygen consumption rate in the cornea $\left(\mathrm{mL}\right.$ of $\mathrm{O}_{2} / \mathrm{cm}^{3}$ of tissue layer /sec), and $t$ is time (seconds).

As we have mentioned, the aerobic metabolism is quantified by the Monod kinetics model, also known as Michaelis Menton Model, ${ }^{5,8}$ which relates the oxygen consumption with the oxygen tension by mean of the expression

$$
Q_{c}\left(p_{c}\right)=\frac{Q_{c, \max } \cdot p_{c}(x)}{K_{m}+p_{c}(x)},
$$


where $K_{m}$ is the Monod dissociation equilibrium constant to which we have referred above. For low oxygen partial pressure $\left(p_{c}<<K_{m}\right)$, the oxygen consumption rate depends linearly on the oxygen tension and tends to zero when the oxygen pressure approaches to zero. For large oxygen pressure $\left(p_{c}>>\right.$ $K_{m}$ ), the consumption also will be dependent on the oxygen tension and it tends to a maximum value when the pressure is equal to $155 \mathrm{~mm} \mathrm{Hg}$ at sea level in the case of open eyes. In such situations, $Q_{c}\left(p_{c}=155 \mathrm{~mm} \mathrm{Hg}\right)=Q_{c}, \max$.

In steady-state conditions, the equations for oxygen transport in the cornea, postlens tear films and lens are, respectively,

$$
\begin{gathered}
\frac{\partial^{2} p_{c}}{\partial x^{2}}=\left(\frac{Q}{D k}\right)_{c} \quad 0 \leq x \leq x_{c} \\
\frac{\partial^{2} p_{\text {tear }}}{\partial x^{2}}=0 \quad x_{c} \leq x \leq x_{c}+x_{\text {tear }}
\end{gathered}
$$

and

$$
\frac{\partial^{2} p_{\text {lens }}}{\partial x^{2}}=0 \quad x_{c}+x_{\text {tear }} \leq x \leq x_{c}+x_{\text {tear }}+x_{\text {lens }},
$$

where $p_{c}, p_{\text {tear }}$ and $p_{\text {lens }}$ represent the partial pressure of oxygen in the cornea, postlens tear film and lens, $D$ is the average diffusion coefficient of oxygen in the cornea tissue $\left(\mathrm{cm}^{2} / \mathrm{sec}\right), k$ is the oxygen solubility coefficient of the cornea tissue (i.e., Henry's law constant) in $\left(\mathrm{cm}^{3}\right.$ of $\mathrm{O}_{2} / \mathrm{cm}^{3}$ layer $/ \mathrm{mm}$ $\mathrm{Hg}), x$ is the distance perpendicular to the surface $(\mathrm{cm}), Q$ is the oxygen consumption rate by the cornea $\left(\mathrm{mL}\right.$ of $\mathrm{O}_{2} / \mathrm{cm}^{3}$ of tissue layer/sec), and $x_{c}, x_{\text {tear }}$, and $x_{\text {lens }}$ are the thicknesses of the cornea, tear film, and lens, respectively.

\section{Solution of the Partial Differential Equation}

This section describes the technical procedure followed using FiPy (available in the public domain at http://www.ctcms.nist. gov/fipy) and a finite volume partial differential equation (PDE) solver using Python software package (Python Software Foundation). ${ }^{36}$ The general equation describing oxygen transport through the lens-tear-corneal system, in one dimension, is Fick's second law with reaction

$$
k(x) \frac{\partial P(t, x)}{\partial t}=k(x) D(x) \frac{\partial^{2} P(t, x)}{\partial x^{2}}-Q[P(t, x)],
$$

where $P_{c}$ is the oxygen partial pressure in the lens-cornea system, $t$ is time, and $x$ is the coordinate normal to the cornea, with $x=0$ at the interface between the anterior chamber and the cornea.

The second term on the right hand side in Equation 1 is the oxygen consumption as a function of the partial pressure, which is absent in the contact lens region and follows a Monod kinetics form in the corneal system:

$$
Q(P)=\frac{Q^{\max } P}{K_{m}+P} .
$$

In Equation 1, the solubility $(k)$ and diffusion coefficient $(D)$ are considered functions of the position, taking constant values across each of the two regions (contact lens and cornea) in the system. Using the above approach we could obtain the complete pressure profile, provided the continuity of the pressure is satisfied in the lens-cornea interface. This is automatically satisfied within our numerical scheme.

We choose standard Dirichlet boundary conditions in the spatial coordinate

$$
P(t, 0)=P_{a c} \quad \text { and } \quad P\left(t, x=L_{c}+L\right)=P_{a i r},
$$

where $P_{a i r}$ is the open eye pressure, corresponding to the atmospheric pressure, and $P_{a c}$ is the oxygen pressure in the anterior chamber.

As for the initial condition, to reproduce the evolution of the pressure profile from the closed eye condition, we need to feed the stationary pressure profile in Equation 1. This stationary closed eye profile can be obtained by solving the steady-state equation

$$
k(x) D(x) \frac{\partial^{2} P_{e s t}(x)}{\partial x^{2}}-Q\left[P_{e s t}(x)\right]=0,
$$

which is obtained from Equation 1 removing the temporal evolution. Equation 4 is subject to the boundary conditions

$$
P_{\text {est }}(0)=P_{a c} \quad \text { and } \quad P_{\text {est }}\left(x=L_{c}+L\right)=P_{P C},
$$

where $P_{P C}$ is the contact-lens/palpebral conjunctiva oxygen pressure.

We then use the solution to Equations 4 and 5 to define

$$
P(0, x)=P_{\text {est }}(x)
$$

as the last boundary condition for Equation 1 .

The system of Equations 4 and 5 and Equations 1 to 3 and 6 are solved using FiPy (Python Software Foundation), a finite volume PDE solver using Python. The Table shows the different values for the parameters used in the numerical solution of the equations. We use a spatial grid with $10^{3}$ points in all computations and time steps of $10^{-1} \mathrm{~s}$ for the timedependent equations.

First Equations 4 and 5 are numerically solved and the resulting profile is used as initial condition for Equations 1 to 3 and 6. An iterative procedure was used due to the nonlinear nature of the transport Equations 1 to 6 , by "sweeping" the solutions over few iterations (see FiPy manual for details). Convergence was reached after the residual was below a predefined value $\left(10^{-11}\right.$ in our case). We checked grid size and time step parameters so that further decrease in size did not result in any improvement. All the computations were performed in a personal computer with an Intel Core i73770K under Debian Linux. Software FiPy version 3.0 was used in all computations.

Multidimensional parameter optimization subject to bounds was done through the "fmin_tnc" function in the Scipy package, which uses a Newton Conjugate-Gradient method. We used this optimization procedure to determine optimized values of the $Q_{\max }$ and $K_{m}$ parameters for a predefined set of the remaining parameters in the model.

\section{References}

1. Fatt I. Steady-state distribution of oxygen and carbon dioxide in the in vivo cornea. II. The open eye in nitrogen and the covered eye. Exp Eye Res. 1968;7:413-430.

2. Brennan NA. A model of oxygen flux through contact lenses. Cornea. 2001;20:104-108.

3. Compan V, Lopez-Alemany A, Riande E, Refojo MF. Biological oxygen apparent transmissibility of hydrogel contact lenses with and without organosilicon moieties. Biomaterials. 2004;25:359-365.

4. Compan V, Tiemblo P, Garcia F, Garcia JM, Guzman J, Riande E. A potentiostatic study of oxygen transport through poly(2-ethoxyethyl methacrylate-co-2,3-dihydroxypropylmethacrylate) hydrogel membranes. Biomaterials. 2005;26: 3783-3791. 
5. Pullum K. The unique role of scleral lenses in contact lens practice. Cont Lens Anterior Eye. 1999;22:S26-S34.

6. Pullum KW, Buckley RJ. A study of 530 patients referred for rigid gas permeable scleral contact lens assessment. Cornea. 1997; 16:612-622.

7. Pullum KW, Whiting MA, Buckley RJ. Scleral contact lenses: the expanding role. Cornea. 2005;24:269-277.

8. Rosenthal P, Croteau A. Fluid-ventilated, gas-permeable scleral contact lens is an effective option for managing severe ocular surface disease and many corneal disorders that would otherwise require penetrating keratoplasty. Eye Contact Lens. 2005;31:130-134.

9. Tan DT, Pullum KW, Buckley RJ. Medical applications of scleral contact lenses: 1 . A retrospective analysis of 343 cases. Cornea. 1995; 14:121-129.

10. Severinsky B, Millodot M. Current applications and efficacy of scleral contact lenses-a retrospective study. J Optom. 2010;3:158-163.

11. van der Worp E, Bornman D, Ferreira DL, Faria-Ribeiro M, Garcia-Porta N, Gonzalez-Meijome JM. Modern scleral contact lenses: a review. Cont Lens Anterior Eye. 2014;37:240-250.

12. Pullum KW, Stapleton FJ. Scleral lens induced corneal swelling: what is the effect of varying Dk and lens thickness? CLAO J. 1997;23:259-263.

13. Smith GT, Mireskandari K, Pullum KW. Corneal swelling with overnight wear of scleral contact lenses. Cornea. 2004; 23:29-34.

14. Efron $\mathrm{N}$. Intersubject variability in corneal swelling response to anoxia. Acta Opbthalmol (Copenb). 1986;64:302-305.

15. Michaud L, van der Worp E, Brazeau D, Warde R, Giasson CJ. Predicting estimates of oxygen transmissibility for scleral lenses. Cont Lens Anterior Eye. 2012;35:266-271.

16. Lopez-Alemany A, Gonzalez-Meijome JM, Almeida JB, Parafita MA, Refojo MF. Oxygen transmissibility of piggyback systems with conventional soft and silicone hydrogel contact lenses. Cornea. 2006;25:214-219.

17. Weissman BA, Ye P. Calculated tear oxygen tension under contact lenses offering resistance in series: piggyback and scleral lenses. Cont Lens Anterior Eye. 2006;29:231-237.

18. Bonanno JA, Stickel T, Nguyen T, et al. Estimation of human corneal oxygen consumption by noninvasive measurement of tear oxygen tension while wearing hydrogel lenses. Invest Ophthalmol Vis Sci. 2002;43:371-376.

19. Wilke CR, Chang P. Correlation of diffusion coefficients in dilute solutions. AICbE J. 1955;1:264-270.

20. Chhabra M, Prausnitz JM, Radke CJ. Diffusion and Monod kinetics to determine in vivo human corneal oxygen-consumption rate during soft contact-lens wear. $J$ Biomed Mater Res B Appl Biomater. 2009;90:202-209.

21. Doughty MJ, Zaman ML. Human corneal thickness and its impact on intraocular pressure measures: a review and metaanalysis approach. Surv Ophthalmol. 2000;44:367-408.

22. Larrea $X$, Buchler P. A transient diffusion model of the cornea for the assessment of oxygen diffusivity and consumption. Invest Ophthalmol Vis Sci. 2009;50:1076-1080.

23. Alvord LA, Hall WJ, Keyes LD, Morgan CF, Winterton LC. Corneal oxygen distribution with contact lens wear. Cornea. 2007;26:654-664.

24. Brennan NA. Beyond flux: total corneal oxygen consumption as an index of corneal oxygenation during contact lens wear. Optom Vis Sci. 2005;82:467-472.

25. Siegfried CJ, Shui YB, Holekamp NM, Bai F, Beebe DC. Oxygen distribution in the human eye: relevance to the etiology of open-angle glaucoma after vitrectomy. Invest Ophthalmol Vis Sci. 2010;51:5731-5738.

26. Harvitt DM, Bonanno JA. Re-evaluation of the oxygen diffusion model for predicting minimum contact lens $\mathrm{Dk} / \mathrm{t}$ values needed to avoid corneal anoxia. Optom Vis Sci. 1999; 76:712-719.

27. Nichols JJ, King-Smith PE. Thickness of the pre- and postcontact lens tear film measured in vivo by interferometry. Invest Ophthalmol Vis Sci. 2003;44:68-77.

28. Ramselaar JA, Boot JP, van Haeringen NJ, van Best JA, Oosterhuis JA. Corneal epithelial permeability after instillation of ophthalmic solutions containing local anaesthetics and preservatives. Curr Eye Res. 1988;7:947-950.

29. Perez JG, Meijome JM, Jalbert I, Sweeney DF, Erickson P. Corneal epithelial thinning profile induced by long-term wear of hydrogel lenses. Cornea. 2003;22:304-307.

30. Haque S, Jones L, Simpson T. Thickness mapping of the cornea and epithelium using optical coherence tomography. Optom Vis Sci. 2008;85:E963-E976.

31. Del-Castillo LF, Ferreira-da-Silva AR, Hernández SI, et al. Diffusion and Monod kinetics model to determine in vivo human corneal oxygen-consumption rate during soft contact lens wear. J Optom. In press. doi:10.1016/j.optom. 2014.06.002.

32. Erickson P, Comstock TL, Zantos SG. Effects of hydrogel lens transmissibility profiles on local corneal swelling during eye closure. Optom Vis Sci. 1996;73:169-177.

33. Holden BA, Mertz GW. Critical oxygen levels to avoid corneal edema for daily and extended wear contact lenses. Invest Ophthalmol Vis Sci. 1984;25:1161-1167.

34. Papas E. On the relationship between soft contact lens oxygen transmissibility and induced limbal hyperaemia. Exp Eye Res. 1998;67:125-131.

35. Fonn D, Du TR, Simpson TL, Vega JA, Situ P, Chalmers RL. Sympathetic swelling response of the control eye to soft lenses in the other eye. Invest Ophthalmol Vis Sci. 1999;40: 3116-3121.

36. Guyer JE, Wheeler D, Warren JA. FiPy: partial differential equations with python. Comp Sci Eng. 2009;11:6-15. 